\title{
THE TECHNOLOGICAL AND FUNCTIONAL VECTORS OF THE KNOWLEDGE SOCIETY
}

\author{
Associate Professor PhD Cornel Nicolae Jucan, \\ University „Lucian Blaga” Sibiu, Romania
}

\begin{abstract}
Since the emergence of human beings appeared as conscious and rational beings, the majority of human actions - including those of economic nature - have been based on knowledge. This interdependence between human actions and knowledge has reflected the stage of human evolution and of the acquired knowledge. What distinguishes the new type of economy from the previous types is the outline and manifestation of knowledge as product, resource, vector and capital, in quasi-decisive proportions, in the functionality and overall performance of the economic and civil society. We hope that the presented elements will highlight new and convincing arguments, which seek to contribute to the speed-up of the construction of the knowledge-based economy in our country.
\end{abstract}

For the creation of the knowledge society, a series of vectors (both technological and functional) must be defined. These vectors should be introduced in a natural succession for the possibilities of our country. In this context, the immediate relation between the information society and the elements of the knowledge society - a relation which requires no successive stages - should be offered special attention with a view to the gradual transformation of the first into the latter. To this end, the careful consideration of the technological and functional vectors which lead to the knowledge society is required.

Examples of vectors of this kind are the following:

\section{Tehnological:}

The Internet, for which a multiple extension is required with regard to the society of knowledge. The necessary factors for this are: the integration of each institution, citizen and residence in this network, broadband transmission (which also means the increase of the speed of transmission) - in relation to this aspect, it should be mentioned that in October 2002 the International Conference of Information Society Technology for Broadband Europe was organised in Bucharest - the transition, supported by the European Union, from the IPv4 protocol to IPv6, the enhancement of those elements connected to contents of the knowledge society, the realization of a system of data and knowledge, the creation of virtual companies and organizations, of virtual museums, etc.

The electronic book, a technology which is already dominant and will become quasiuniversal. SOFTWIN Company has already approached this field on an international scale. The Romanian Academy, together with the Central Institute of Informatics (ICI), SOFTWIN and the Romanian Ministry of Education and Research organized in 2001 an international conference, in which the role of the electronic book in the Romanian economy and culture was emphasized for the first time in our country. In addition, a volume with the papers presented at the conference was published and ICI founded an electronic library, at the initiative of Prof. Doina Banciu.

Unfortunately, the activity of this library cannot be carried out in a natural way in our country, as it is carried out in other countries. A certain production of electronic books began in Romania, but the most part of it is rather of a "decorative nature".

Artificial intelligence, in connection to which we support the following statements of the experts: "under crew conditions, knowledge society is based on intelligent agents in the majority of socio-economical activities. The artificial intelligence represents the technological essence of the 
knowledge society. It unites and stimulates the internet, nanotechnologies, and the functional vectors of the knowledge society. Artificial Intelligence in the first 20 years of the twenty-first century will surpass human intelligence (as far as the structural aspects are concerned, not in relation to intuition and creativity)".

Gradually, all the informational technologies resort to intelligent agents. Intelligent agents, who are essentially knowledge based systems with artificial intelligence, are used both for data mining and for knowledge discovery. The human being will increasingly carry out his activity - in our country as well - in an intelligent environment.

The basis of our assertions is the fact that the Romanian research in the field of artificial intelligence began in 1980 at the Central Institute of Informatics, and, in 1981, the first national conference on artificial intelligence was organized by the same institute. In addition, concrete products were realized by the Gheorghe Tecuci Academy (the DISCIPOL system), later developed at Mason University in the US by Dan Tufiş, correspondent member of the Romanian Academy and the director of the Institute of Research for Artificial Intelligence. In April 2004, Dan Tufiş also organized, after more than twenty years, the second national conference of Artificial Intelligence. Another significant event took place in Iasi from the 15 to the 20 of July 2002. The European Conference on Intelligent systems and Technologies was organized under the leadership of prof. Nicolaie Teodorescu, correspondent member of the Romanian Academy.

Nanoelectronics, which will probably become the main physical support not only for the processing of information, but also for many other functions of the knowledge society within a decade. The National Institute of Microtechnology (acad. Dan Dascălu, Mihai Mihăilă, correspondent member of the Romanian Academy, and dr. Irina Kleps) has founded a Research Center for Nanotechnologies, whose activity is constantly being developed.

\section{Functional:}

The Management of knowledge will become the basic functional activity in all the levels of the knowledge society for companies, organisations, institutions, local and national administrations. A significant aspect in this respect will be that of the moral utilization of knowledge at a global level. This aspect will become a real concern for the future knowledge society.

E-learning, the second fundamental functional activity. In this context, the Romanian Academy, in collaboration with SOFTWIN, with the Romanian Ministry of Education and Research, and with SIVECO, organised in 2002 a conference dedicated to the evaluation of the present state and of the perspectives of this functional vector in Romania.

E-health, the system of care at a social and individual level, at which the study of the genome and of the bioinformatics of genes and of biological proteins with regard to the functions determined by the gene structures is added.

E-government, to which a considerable attention has been given both at a national and at a European level, but which will have to be reconsidered for the knowledge society.

E-economy, which will be transformed in a knowledge-based economy, with various specific aspects.

The protection of the environment and the insurance of a durable society, which will only be possible through the knowledge society.

Some other elements are: the thoroughgoing study of human existence, the development of new technological knowledge, the development of a society based on knowledge and innovation, etc.

\section{Perspectives}

An analysis of the situation of the technology of information in Romania reveals a significant effort, which ensures that tradition and cultural atmosphere favourable for the growth and development of the information and knowledge society. However, we can observe that in 1999 Romania was a rather undeveloped country from the informational point of view. Even Varujan 
Pambuccian, the president of the expert commission for the information technology and telecommunications of the Parliament of Romania drew attention, at the beginning of 2001, to the presence of two informational side of Romanian: an advanced, yet limited, side, and another utterly underdeveloped one (the underdeveloped one being prevalent).

Nevertheless, our perspectives have become optimistic. A study shows that, beginning with 2002, over 60 percent of the students in Bucharest had a personal computer at home.

A series of governmental actions and achievements of private companies and of educational and research institutions give us confidence that we are actually "catching up" with the European information society.

The problem that must be urgently solved is the need of immediate action in order to keep pace with the knowledge society. Moreover, this society must be connected as soon as possible to the requirements of the knowledge based economy.

In Romania, the research activity in the field of artificial intelligence is still, unfortunately, in an incipient phase. The majority of the intelligent systems are imported together with the techniques whose component parts they are.

The expert systems, which assist the economic-financial decision, are prevalently used in the financial, banking, and insurance institutions. The transition period and the lack of a powerful competition on the Romanian market cause the absence of such institutions at a company level. In many of the economic institutions in our country the economic activity is orientated by the intuition of the manager. With the accentuation of competition, when the profit of the company mainly depends on the marketing and management activities used - and this will resist on the economic markets only if it will be able to optimize its activity - the necessity of the expert systems becomes indispensable. The expert systems will assist the economic-financial decisions of the managers and of the enterprisers with a view to solving a given situation with prompt solutions and advice and in real time. The importance and profitableness of using the expert systems is not only a consequence of the low prices needed for their usage, but also of the promptness and efficiency of the decisions provided by the expert systems in relation to the performed economic activity.

The advantages of the expert systems ${ }^{1}$ both in the economic-financial field and in other fields of activity are numerous, varying in accordance to the type of field in which they are applied.

1. The expert systems are a "treasure of knowledge", integrating the work and experience of the experts in the field. This knowledge is available permanently and without additional charges to the consumer, and it must be periodically brought up-to-date, according to the latest modifications and improvements.

2. The expert system can provide decisions, solutions, advice, etc., within a very short space of time, thereby significantly simplifying and reducing the process of decision.

3. The utilization of the expert system does not require high qualifications in the specific field, but the results of its utilization may attain the level of the results yielded by the human expert in the respective field.

4. The expert system can be an excellent guidance for the consumer due to the manner in which it explains the obtained results on the basis of the employed principles.

5. The costs of an expert system as related to its benefits are definitely advantageous in comparison to the wages expenses and other incentives paid to the human experts.

6. Furthermore, an expert system also has the advantage of being easily maintained and easily developed with the aid of expert system generators. Another benefit of the expert system is its high portability and undemanding documentation.

\footnotetext{
${ }^{1}$ Ioan Andone, Alexandru Țugui, Intelligent systems in management, book-keeping, banking, finance, and marketing, The Economic Publishing House, Bucharest, 1999, p.71, 72. ${ }^{1}$
} 
At the same time, the benefits brought by the implementation of an expert system in a company are also worth mentioning :

1. Rationing effects:

- Economies based on the costs of the personnel;

- The better utilization of the capacities of production;

- Savings resulted from production;

- Savings resulted from automated documentation.

1. Effects for quality improvement:

- The exploitation of the effects from multiple rationalisations;

- The control of the solutions proposed by the personnel and the programs;

- High qualification and efficient training of the personnel;

- The rapid and better design of the offers with a view to an increase in orders;

- The equipment of expensive products with intelligence for the same level of diagnosis;

- The automatization of the activities of the company with a view to the improvement of the manufacturing networks.

2. Positive organizational effects:

- The multiplication of the expert examination;

- The conservation of the expert examination;

- The decentralization of decision making;

- The expansion of the exchange and evolution of the expert examination;

- The dynamic distribution of decisions.

\section{Bibliography:}

Bogdan I. et. al

Jucan Cornel Nicolae

Jucan Cornel Nicolae

Jucan Cornel Nicolae , Ciontu Sorin

Nicolaescu O. şi Nicolaescu L.

Zaharie D şi colectiv
Treatise of Banking and Financial Management, The Economic Publishing House, Bucharest, 2002;

Expert systems in the creation of financial decisions, Alma Mater Publishing House, Sibiu, VOL I. /2003; VOL.II /2006.

Expert systems, reflections in the national economy, Alma Mater Publishing House, Sibiu, 2002;

Information Systems for the assistance of the financial decision, Alma Mater Publishing House, 2004;

The Economy, the company, and the Management based on knowledge, The Economic Publishing House, Bucharest, 2005;

Expert Systems - theories and applications, Dual Tech Publishing House, Bucharest, 1999 